\title{
The X-Ray AGN Bias Factor at Zero Redshift
}

\author{
Roman A. Krivonos ${ }^{1,2}$ and Mikhail G. Revnivtsev ${ }^{2,3}$ \\ ${ }^{1}$ Max Planck Institute for Astrophysics, Garching, Germany \\ Email: krivonos@mpa-garching.mpg.de \\ ${ }^{2}$ Space Research Institute, Moscow, Russia \\ ${ }^{3}$ Excellence Cluster Universe, Technische Universitat München, Garching, Germany
}

\begin{abstract}
Using an AGN sample from the INTEGRAL all-sky survey, we show that the density fluctuations of matter in the local universe translate linearly into the density fluctuations of accreting supermassive black holes. Our results imply that hard X-ray emitting AGNs are unbiased tracers of the galaxy population in the nearby universe and SMBH activity is independent of the density of galaxies.
\end{abstract}

Keywords. large-scale structure of universe, galaxies: Seyfert, X-rays: galaxies

We have studied the volume density variations of nearby $(D<70 \mathrm{Mpc})$ hard X-ray emitting AGNs from the INTEGRAL all-sky survey conducted by Krivonos et al. (2007). AGN clustering is characterized by a so-called bias factor, i.e., ratio of fractional X-ray AGN-density fluctuations to the fractional matter-density fluctuations. We used the most straightforward way to measure the AGN bias factor by directly correlating the matterdensity variations with the AGN volume density. As a tracer of the matter distribution, we use the IRAS PSCz redshift survey of Saunders et al. (2000). Volume-density fluctuations of IRAS galaxies in the local universe reflect matter-density fluctuations, i.e., their bias factor approachs unity (Basilakos \& Plionis 2006). We calculated the volume-density excess for different directions in the sky by using 39 X-ray AGNs and 3680 IRAS galaxies at $D<70 \mathrm{Mpc}$ (for details see Krivonos et al. 2007). X-ray and IR density fluctuations are related by dependence that is consistent with a linear law. Our preliminary best-fit value of the IR to $\mathrm{X}$-ray ratio is $0.80 \pm 0.13$.

The measured X-ray AGN bias factor is consistent with unity, which means that matter-density fluctuations on a scale of $70 \mathrm{Mpc}$ at zero redshift translate linearly into fluctuations of the X-ray AGN volume-density. Hard X-ray emitting AGNs are apparently unbiased tracers of the galaxy population in the nearby universe. The measured AGN bias factor implies that SMBH activity is independent of the density of galaxies on spatial scales of $70 \mathrm{Mpc}$.

\section{Acknowledgements}

This work was supported by grants of the RFBR (08-08-13734, 08-02-00974, NSh5579.2008.2) and programs of Presidium of RAS P04 and OFN-17.

\section{References}

Basilakos, S. \& Plionis, M. 2006, MNRAS, 373, 1112

Krivonos, R., et al. 2007, A\& A, 475, 775

Saunders, W., et al. 2000, MNRAS, 317, 55 